\title{
Research
}

\section{Antisperm antibodies: Incidence, isotypes and location on spermatozoa, their implications on fertilization and on pregnancy rate at a selected centre in Sri Lanka}

\author{
Varuni Tennakoon ${ }^{1}$ Surangi G Yasawardene ${ }^{2}$ Deepal S Weerasekera $^{3}$ \\ Sri Lanka Journal of Obstetrics and Gynaecology 2010; 32: 8-16
}

\begin{abstract}
Objectives: 1. To investigate the incidence, isotypes and location of antisperm antibodies (ASA) on the sperm surface in an infertile population. 2. To study the influence of isotypes, location of ASA on the sperm surface on fertilization, cleavage rates of embryos and pregnancy rate.
\end{abstract}

Methods: A prospective analytical study was carried out on infertile couples who underwent Assisted Reproductive Technology (ART) procedures at 'Prarthana' Centre for in vitro fertilization (IVF) during three year period from 01.01.2006 - 01.01.2009. Presence of ASA was elicited using mixed agglutination reaction latex bead test (Sperm MAR, Fertipro NV, Belgium) in male and female subjects. The isotype and location of ASA, detected by the type (i.e. $\operatorname{IgA}, \operatorname{IgG}$ ) and the site of binding of latex beads to ASA on the spermatozoa (i.e. head, midpiece, tail of the sperm) was analyzed. In females who underwent In-vitro fertilization, fertilization rate and day 03 cleavage rate of embryos were assessed. The pregnancy rates following each ART procedure were noted. A total of 230 infertile couples were investigated.

Results: Out of 230 couples, 48 (20.86\%) were positive for ASA. Among ASA positives, 19 (39.58\%), 13 (27.08\%), 16 (33.34\%) had IgA, IgG, both IgA, IgG respectively and 10 (20.83\%), 24 (50\%), 11 (22.92\%), 03 (6.25\%) had head, tail, midpiece+tail, whole sperm bound ASA

${ }^{1,3}$ Prarthana Centre for ART, Rajagiriya, Colombo.

2 Dept. of Anatomy, Faculty of Medical Sciences, University of Sri Jayawardenepura, Gangodawila, Nugegoda.

Correspondence: Varuni Tennakoon

E-mail:varuni16email@yahoo.com respectively. The total fertilization rate and cleavage rate among ASA positives were $69.38 \%(179 / 258)$ and 53.63\% (96/179) respectively; and among ASA negatives the rates were $58.54 \%(473 / 808)$ and $61.73 \%(292 / 473)$ respectively. Couples with IgA, IgG and IgA+IgG achieved a pregnancy rate of $10.53 \%$, $23.08 \%$ and $18.75 \%$ respectively. Out of 08 pregnancies $05,02,01$ had ASA on tail, midpiece+tail and whole sperm respectively.

Conclusions: It was found that presence of ASA has negative effects on fertilization and post fertilization events. IgA isotype of ASA demonstrated the lowest pregnancy rate. When majority of sperms are coated with ASA on the heads or mid piece+tail, cleavage rates were often impaired. Intra-cytoplasmic sperm injection is recommended for such couples to achieve a successful pregnancy.

Key words: Antisperm antibodies, incidence, isotypes, binding site, in vitro fertilization, intracytoplasmic injection, pregnancy rate.

\section{Introduction}

Research in antisperm antibodies (ASA) began in 1899 when Landsteiner initially reported that sperm could be antigenic if injected into a foreign species. Since then ASA in infertility has been largely investigated and has led to the possibility that naturally occurring male and female ASA could serve as one mechanism for human infertility ${ }^{1}$. The incidence of ASA has been estimated to range from $9 \%-36 \%$ in infertile couples depending on the test format, detecting assays and the reporting centre ${ }^{2}$. ASA have been theorized to negatively impact fertility by affecting sperm motility, cervical mucus penetration, gamete fusion and potentially even the first steps of embryo development ${ }^{2}$. These antibodies present within the reproductive tract may be both transudates from the blood or secreted locally by submucosal plasmacells ${ }^{3}$. Developmental abnormalities of the formation of the blood-testis barrier and its traumatic disruption could 
lead to ASA formation in males ${ }^{3,4}$. In females mechanical or chemical disruption of the mucosal layer of the female genital tract may permit exposure to foreign sperm antigens and ultimately formation of $\mathrm{ASA}^{5}$. Women undergoing repeated intra uterine insemination (IUI) with washed spermatozoa, are also thought to be prone to develop $\mathrm{ASA}^{6}$. Removal of immnosuppressive properties of seminal plasma when preparing the semen for IUI could be an added factor ${ }^{7}$. There are three major structural types of antisperm immunoglobulins: IgA, IgG and IgM; each of the three types can be bound to the whole sperm surface or selectively to the head, midpiece or tail of the spermatozoa when assessed by the latex bead technique. IgM is a larger molecule and rarely present in semen or cervical mucus. Also it is rarely detected alone or combined with IgA/IgG. Therefore it is generally considered as having less clinical importance.

The aims of this study were,

1. To find out the incidence of ASA among infertile couples in a Sri Lankan set up.

2. To study the effect of ASA on fertilization, cleavage rate of embryos and clinical pregnancy rate comparing ASA positive and negative couples.

3. To study the influence of isotype and topographical location of ASA on fertilization, cleavage and clinical pregnancy rate of ASA positive couples.

4. To demonstrate whether intracytoplasmic sperm injection (ICSI) is preferable to standard IVF (in vitro fertilization) as the method of oocyte insemination in certain isotypes and locations of ASA in ASA positive couples.

This study also compares the findings with those already published in the literature and provides valuable insights for the investigation and management of infertile couples in Sri Lanka.

\section{Materials and methods}

This prospective analytical study was conducted on infertile couples undergoing ART (assisted reproductive technology) procedures at 'Prarthana', Centre for IVF, 1175, Cotta Road, Rajagiriya, from 01.01.2006 to 01.01.2009. All infertile couples who consented (informed written consent was taken) and who was seeking treatment at 'Prarthana', Centre for IVF were recruited for the study. The total study population was 230 infertile couples; 460 individuals.
The ethical clearance for the study was obtained from the Ethical Review Committee of Faculty of Medical Sciences, University of Sri Jayawardenepura, Gangodawila, Nugegoda, Sri Lanka.

Each couple underwent a single cycle of ART procedure i.e. either IUI or IVF-ET (embryo transfer) as the method of treatment for infertility. The method of treatment for each couple was decided depending on other factors such as age, duration of infertility, previous failed treatment procedures etc. other than presence of ASA. In the male partner spermatozoa, seminal plasma and serum were examined for ASA isotypes of IgA and IgG. In the female partner, cervical mucus and serum were examined for both ASA isotypes. In females who underwent IVF, follicular fluid was also examined for ASA-IgA and IgG.

The ASA were detected using mixed agglutination reaction latex bead test (SpermMAR, Fertipro $\mathrm{NV}$, Belgium). In mixed agglutination reaction (MAR) assay, blood group $\mathrm{O} \mathrm{Rh}$ positive erythrocytes are coated with human $\operatorname{IgG}$ or $\operatorname{IgA}$ and are subsequently mixed with washed or unwashed, viable sperms. The sperm erythrocyte agglutination in the presence of ASA is observed by light microscopy. Instead of erythrocytes coated with immunoglobulin, the 'SpermMAR' test, which is a commercially available test, have IgA and IgG coated latex particles to detect ASA. This test provides a rapid assay time, good specificity, isotype and the location of the ASA and the ability to use viable sperms ${ }^{8}$.

The direct 'SpermMAR' test was performed on spermatozoa and indirect test was performed on seminal plasma, cervical mucus, serum and follicular fluid to elicit ASA. The percentage of motile sperm exhibiting latex bead binding was calculated. A test with $>30 \%$ of the sperms with bound beads was considered 'positive for ASA' (WHO laboratory guidelines ${ }^{27}$ ). The isotype of the ASA was observed. Furthermore, the site of attachment was also considered: binding to the head, midpiece, tail or to the whole sperm as well as to more than one region was indicated.

Washed sperm samples were used for both IUI and IVF. In couples that underwent IVF, the preovulatory oocytes in the metaphase II and late metaphase I stages of development were considered in determining the fertilization rate. Oocytes were inseminated either by standard IVF or intracytoplasmic sperm injection (ICSI). In standard IVF a drop of sperms containing $1 \times 106-2 \times 106 \mathrm{mil} / \mathrm{ml}$ sperms was placed aside of the oocyte in-vitro. In ICSI a single sperm was manually injected into the cytoplasm of the oocyte. 
Oocytes were observed 18-24 hours following insemination by standard IVF or ICSI under the inverted microscope. Fertilization of the oocyte by the sperm was confirmed, if the cell had 02 pronuclei and 02 polar bodies. Fertilization rate was assessed as,

Fertilization rate $=$

total no of fertilized oocytes $\times 100$

total number of inseminated oocytes.

Day 03 cleavage of the embryo was observed under the inverted microscope. Embryos with 6-8 evenly placed cells with no or mild to moderate fragmentation and cytoplasmic granularity were considered as well cleaved embryos. Cleavage rate was assessed as,

\section{Cleavage rate $=$}

no of good quality D3 embryos $\times 100$

total number of fertilized oocytes at day 01.

Following each ART procedure the clinical pregnancies were noted. The clinical pregnancy was confirmed at 08 weeks of gestation by an ultra sound scan revealing a foetus with heart beat. Clinical pregnancy rate was assessed as,

Clinical pregnancy rate $=$

total number of clinical pregnancies $\times 100$

Total number of embryo transfers

Two sample $\mathrm{z}$ test and Fisher's exact test were used to analyze statistical significance either with two categories or with ASA negative category. ANOVA was used to analyze data in more than two categories in this study. The fertilization and cleavage rates of ASA negative category were taken as the standard to compare with the ASA positive category.

\section{Results}

\section{Incidence of antisperm antibodies}

The incidence of antisperm antibodies among the infertile couples of the study sample was $20.87 \%$ ( $n=48$, $48 / 230$ ). Antisperm antibody incidence among the males in the sample was $12.61 \%(n=29,29 / 230)$ while that for the females was $8.26 \%(n=19,19 / 230)$.

\section{Effect of ASA on fertilization rate, cleavage rate and clinical pregnancy rate in ASA positives and ASA negatives}

Figure 1 shows the number of couples underwent each procedure, number of clinical pregnancies achieved in each procedure and total number of inseminated oocytes, number of fertilized oocytes and number of cleaved embryos in those who underwent IVF. Table 1 shows the fertilization and cleavage rate of embryos among ASA positives and negatives in IVF. It was observed that in ASA positives the total fertilization rate $(69.38 \%$ both standard IVF and ICSI inclusive) was significantly higher $(P$-value $=0.001)$ than that of the ASA negatives (58.54\%). When comparing the total cleavage rates, it was observed that ASA positives $(53.63 \%)$ had a significantly lower $(P$-value $=0.037)$ cleavage rate than that of ASA negatives $(61.73 \%)$. Therefore, this data shows that although higher fertilization rates are seen in ASA positives, the cleavage rates are significantly lower than the ASA negatives, supporting the fact that ASA have negative effects on post fertilization events.

Among ASA positives, it was observed a proportionately higher clinical pregnancy rate in those who underwent IVF (19.23\%) than those who underwent IUI (13.64\%). Among ASA negatives the clinical pregnancy rates were $22.54 \%$ in IVF and $20.24 \%$ in IUI. Statistical significance was not calculated here due to small sample size.

The occurrence of immunoglobulin (Ig) isotypes of ASA

Among ASA positives, 19 (39.58\%), 13 (27.08\%) and $16(33.34 \%)$ demonstrated $\operatorname{IgA}, \operatorname{IgG}$ and both IgA+IgG respectively.

\section{Impact of Ig isotypes of ASA on fertilization rate and cleavage rate}

Table 2 shows the fertilization and cleavage rates corresponding to the Ig isotypes of ASA. Among Ig isotypes it was observed that there was no significant difference in fertilization rates and cleavage rates when oocytes undergo standard IVF or ICSI. Hence it shows that $\mathrm{Ig}$ isotypes do not have an impact on method of insemination of oocytes. Irrespective of the method of insemination, the total fertilization rate of oocytes were higher with IgA $(82.76 \%)$ and $\operatorname{IgG}(70.75 \%)$ compared to that of ASA negatives (58.54\%). The cleavage rates were markedly low in IgA (45.82\%) and IgG (49.33\%) compared to that of ASA negatives (61.73\%), though there is no statistically significant difference observed. In IgA+IgG, the total fertilization rate $(65.04 \%)$ and cleavage rate $(60 \%)$ differ marginally with those of ASA negatives. IgA isotype was observed as having the highest fertilization rate and the lowest cleavage rate. 
Figure 1

Flow chart of the study group
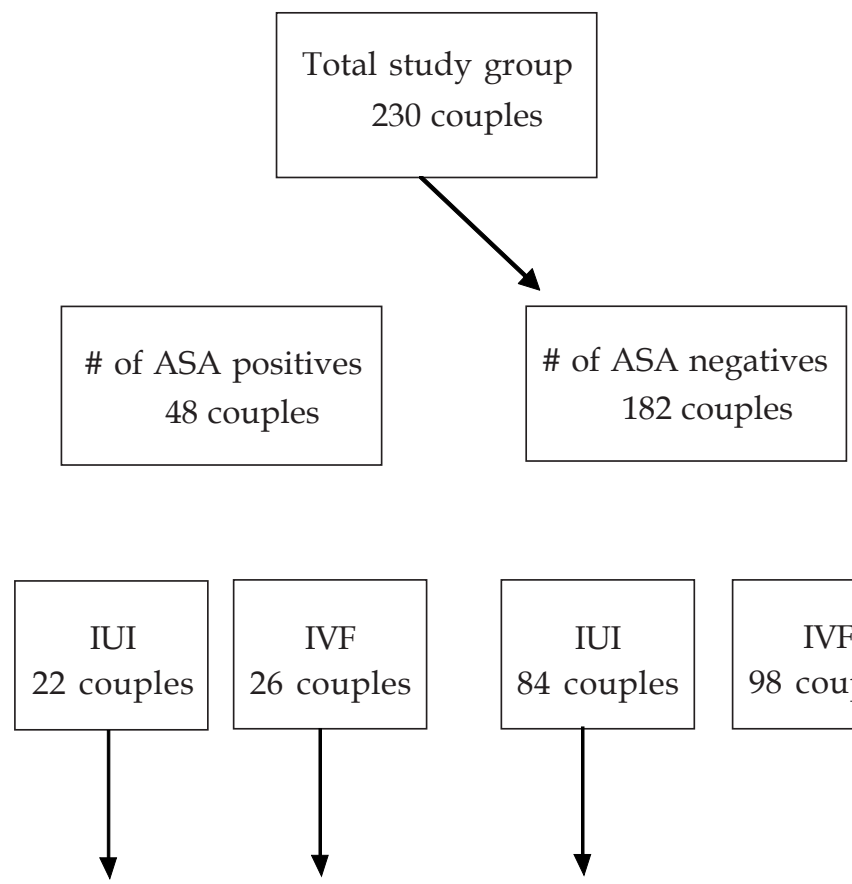

IVF

98 couples

Number of oocytes inseminated

258

808

Number of oocytes fertilized

179

473

Number of oocytes cleaved

96

292

Number of inseminations/embryo transfers

22

26

84

98

Number of clinical pregnancie

03

05

17

22

Table 1. Fertilization rates and cleavage rates of embryos among ASA negatives and ASA positives

\begin{tabular}{|c|c|c|c|c|c|c|}
\hline & \multirow{2}{*}{$\begin{array}{c}\text { Total } \\
\text { fertilization } \\
\text { rate }(\%)\end{array}$} & \multirow{2}{*}{$\begin{array}{l}\text { Total } \\
\text { cleavage } \\
\text { rate }(\%)\end{array}$} & \multicolumn{2}{|c|}{ Standard IVF } & \multicolumn{2}{|c|}{ ICSI } \\
\hline & & & $\begin{array}{c}\text { Fertilization } \\
\text { rate }(\%)\end{array}$ & $\begin{array}{l}\text { Cleavage } \\
\text { rate }(\%)\end{array}$ & $\begin{array}{c}\text { Fertilization } \\
\text { rate }(\%)\end{array}$ & $\begin{array}{l}\text { Cleavage } \\
\text { rate }(\%)\end{array}$ \\
\hline $\begin{array}{c}\text { ASA } \\
\text { negatives }\end{array}$ & $\begin{array}{c}58.54 \\
(473 / 808)\end{array}$ & $\begin{array}{c}61.73 \\
(292 / 473)\end{array}$ & $\begin{array}{c}61.38 \\
(213 / 347)\end{array}$ & $\begin{array}{c}64.32 \\
(137 / 213)\end{array}$ & $\begin{array}{c}56.4 \\
(260 / 461)\end{array}$ & $\begin{array}{c}59.62 \\
(155 / 260)\end{array}$ \\
\hline $\begin{array}{c}\text { ASA } \\
\text { positives }\end{array}$ & $\begin{array}{c}69.38 \\
(179 / 258)\end{array}$ & $\begin{array}{c}53.63 \\
(96 / 179)\end{array}$ & $\begin{array}{c}73.33 \\
(55 / 75)\end{array}$ & $\begin{array}{c}56.36 \\
(31 / 55)\end{array}$ & $\begin{array}{c}67.76 \\
(124 / 183)\end{array}$ & $\begin{array}{c}52.42 \\
(65 / 124)\end{array}$ \\
\hline
\end{tabular}


Table 2. Immunoglobulin (Ig) isotype of ASA and corresponding fertilization rates and cleavage rates of embryos

\begin{tabular}{|c|c|c|c|c|c|c|}
\hline \multirow{2}{*}{$\begin{array}{l}\text { Isotype } \\
\text { of Ig }\end{array}$} & \multirow{2}{*}{$\begin{array}{c}\text { Total } \\
\text { fertilization } \\
\text { rate }(\%)\end{array}$} & \multirow{2}{*}{$\begin{array}{l}\text { Total } \\
\text { cleavage } \\
\text { rate (\%) }\end{array}$} & \multicolumn{2}{|c|}{ Standard IVF } & \multicolumn{2}{|c|}{ ICSI } \\
\hline & & & $\begin{array}{c}\text { Fertilization } \\
\text { rate }(\%)\end{array}$ & $\begin{array}{l}\text { Cleavage } \\
\text { rate (\%) }\end{array}$ & $\begin{array}{c}\text { Fertilization } \\
\text { rate }(\%)\end{array}$ & $\begin{array}{c}\text { Cleavage } \\
\text { rate (\%) }\end{array}$ \\
\hline IgA & $\begin{array}{l}82.76 \\
(24 / 29)\end{array}$ & $\begin{array}{l}45.83 \\
(11 / 24)\end{array}$ & $\begin{array}{c}75 \\
(6 / 8)\end{array}$ & $\begin{array}{c}50 \\
(3 / 6)\end{array}$ & $\begin{array}{c}85.71 \\
(18 / 21)\end{array}$ & $\begin{array}{c}44.4 \\
(8 / 18)\end{array}$ \\
\hline IgG & $\begin{array}{c}70.75 \\
(75 / 106)\end{array}$ & $\begin{array}{c}49.33 \\
(37 / 75)\end{array}$ & $\begin{array}{c}77.77 \\
(28 / 36)\end{array}$ & $\begin{array}{c}46.43 \\
(13 / 28)\end{array}$ & $\begin{array}{c}67.14 \\
(47 / 70)\end{array}$ & $\begin{array}{c}51.06 \\
(24 / 47)\end{array}$ \\
\hline $\operatorname{Ig} A+\operatorname{IgG}$ & $\begin{array}{c}65.04 \\
(80 / 123)\end{array}$ & $\begin{array}{c}60 \\
(48 / 80)\end{array}$ & $\begin{array}{c}67.74 \\
(21 / 31)\end{array}$ & $\begin{array}{c}71.43 \\
(15 / 21)\end{array}$ & $\begin{array}{c}64.13 \\
(59 / 92)\end{array}$ & $\begin{array}{c}55.93 \\
(33 / 59)\end{array}$ \\
\hline
\end{tabular}

\section{Ig isotypes of ASA with clinical pregnancies}

A total of 08 clinical pregnancies were observed among the ASA positive (3-IUI, 5-IVF) couples, following the ART procedure. Table 3 shows the Ig isotypes of ASA with the number of pregnancies. It was observed that couples with IgG isotype of ASA achieved the highest pregnancy rate $(23.08 \%)$ while couples with IgA isotype of ASA had the lowest pregnancy rate $(10.53 \%)$.

Table 3. Ig isotype of ASA in ASA positive couples and corresponding number of pregnancies

\begin{tabular}{llll}
\hline $\operatorname{Ig} A$ & $\operatorname{Ig} G$ & $\operatorname{Ig} A+\operatorname{IgG}$ \\
\hline
\end{tabular}

Number of

ASA

positive

couples

19

13

16

Number of

clinical

pregnancies

$2(10.53 \%) \quad 3(23.08 \%) \quad 3(18.75 \%)$

\section{Location of ASA on spermatozoa}

Among ASA positives, 10 (20.83\%), 24 (50\%), 11 $(22.92 \%), 03(6.25 \%)$ had head, tail, midpiece+tail and whole sperm bound ASA respectively. The data shows that $50 \%$ of the ASA positives had ASA bound to the tail of the sperm.

\section{Impact of the location of ASA on fertilization}

Table 4 shows the fertilization and cleavage rates in standard IVF and ICSI corresponding to location of ASA among couples that underwent IVF. It was observed that in standard IVF the cleavage rates (52.63\% and $37.5 \%$ respectively) were markedly lower when ASA was located around the head and significantly lower $(\mathrm{P}$-value $=0.03$ ) when located on midpiece+tail of the spermatozoa compared to the cleavage rate $(64.34 \%)$ in ASA negatives. When ASA was located around the tail of spermatozoa, there was no significant difference observed in cleavage rate compared to that of ASA negatives. It was also observed that head bound ASA oocytes had a markedly lower $(52.63 \%)$ and midpiece+tail bound ASA oocytes $(37.5 \%)$ had a significantly lower $(P$-value $=0.026)$ cleavage rate than that of the tail bound ASA oocytes (75\%).When oocytes were inseminated by ICSI, there was no statistically significant 
difference observed in cleavage rates of head $(50.88 \%)$, tail $(55.32 \%)$ and midpiece+tail $(41.18 \%)$ bound ASA than that of ASA negatives (59.62\%), except the whole sperm bound ASA. The whole sperm bound ASA had few numbers of oocytes which made statistical analysis impossible for that category. The differences of cleavage rates between binding sites were more or less alleviated by ICSI over standard IVF as the method of insemination. Therefore this data demonstrates that head or midpiece+tail bound ASA on spermatozoa exhibit more negative effects on cleavage rate in standard IVF. However, when ICSI was performed on oocytes, the negative effects of head and midpiece+tail bound ASA on cleavage of embryos were suppressed.

\section{Location of ASA with clinical pregnancies}

Table 5 shows the location of ASA with the number of pregnancies. Most number of pregnancies was observed when ASA was bound to the tail of the spermatozoa. There were no pregnancies achieved when ASA was bound to the head of the spermatozoa. However, one pregnancy was achieved with ASA bound to whole surface of spermatozoa by performing ICSI as the method of fertilization.

\section{Table 4. Location of ASA on spermatozoa and corresponding fertilization rate and cleavage rate of embryos in standard IVF and ICSI}

Site of binding

$\begin{array}{cccc}\text { Fertilization } & \text { Cleavage } & \text { Fertilization } & \text { Cleavage } \\ \text { rate }(\%) & \text { rate }(\%) & \text { rate }(\%) & \text { rate }(\%)\end{array}$

$\begin{array}{lcrrr}\text { Head } & 70.37(19 / 27) & 52.63(10 / 19) & 67.86(57 / 84) & 50.88(29 / 57) \\ \text { Tail } & 68.97(20 / 29) & 75(15 / 20) & 64.38(47 / 73) & 55.32(26 / 47) \\ \text { Midpiece+tail } & 84.21(16 / 19) & 37.5(6 / 16) & 85(17 / 20) & 41.18(07 / 17) \\ \text { Whole sperm } & - & - & 50(03 / 06) & 100(03 / 03)\end{array}$

Table 5. Location of ASA in ASA positive couples and corresponding pregnancies

\begin{tabular}{lcccc}
\hline & Head & Tail & Midpiece+tail & Whole sperm \\
\hline Number of ASA positive couples & 10 & 24 & 11 & 3 \\
Number of clinical pregnancies & - & 5 (3-IUI, & 2 (1-Std IVF, & 1-(ICSI) \\
\hline
\end{tabular}




\section{Discussion}

Sperms have been known to be antigenic for more than a century. Although there is a strong body of evidence that in humans and in other species at least some antibodies that bind to sperm antigens can cause infertility, it is still a debated matter. This is mainly due to the fact that a significant percentage of fertile couples have detectable ASA. This clearly shows that these antibodies do not always disrupt fertility. Investigations of ASA and improvements in their methods of detection have advanced rapidly during the 1970s, 1980s and early 1990s but not so much during the last few years. This is in large part due to the observation that ICSI could be used as an effective treatment for circumventing immunoinfertility ${ }^{8}$. Most existing techniques used to detect ASA, fall short of being able to distinguish reliably between ASA that contribute to infertility and those that do not ${ }^{11}$. However, the rational use of current ASA-tests can be effective in screening and quantifying sperm autoimmunization relevant to infertility ${ }^{9}$.

Analysis of fertilization data from IVF programs provides a direct means of assessing the possible effects of antisperm antibodies on human fertilization processes. The relatively small numbers of patients with ASA found in any individual IVF program invariably makes statistical analysis difficult and also makes it difficult to compare groups of patients. Meanwhile the direct and indirect latex bead test is of particular value in evaluating the different isotypes of $\operatorname{Igs}(\operatorname{Ig} A, \operatorname{IgG}, \operatorname{Ig} M)$ and their distribution on various regions of the sperm surface (head, midpiece, tail) ${ }^{10,11}$. Several studies have demonstrated low fertilization and cleavage rates of embryos in ASA positives. Vazquez-Levin in 1997 demonstrated that fertilization and early embryonic cleavage found to be reduced significantly in ASA positives ${ }^{12}$. Most of the recent studies also show the negative effects of ASA on fertilization and cleavage rates ${ }^{13,14,15}$. In contrast, few other authors found that ASA did not affect spermoocyte binding ${ }^{16}$.

In this study, fertilization was not affected due to the presence of ASA. In fact ASA positives showed a significantly higher fertilization rate $(P$-value $=0.001)$ than ASA negatives. However, the cleavage rate of embryos in ASA positives was significantly lower $(P$-value $=0.037)$ than that of ASA negatives. Therefore our data indicate that the antibodies to sperm surface antigens may inhibit early cleavage of oocytes although it does not affect fertilization. These findings may suggest that these antigens may constitute an extra nuclear cleavage signal for early division of fertilized zygotes ${ }^{17}$. Extensive studies involving many IVF centres are in need to investigate the magnitude of this effect.
When evaluating the relationship between Ig isotype of ASA and fertilization, Witkin et al., found that sperm bound antibodies, mainly IgA may directly lower the fertilization rate ${ }^{18}$. Other studies have shown the influence of isotype of ASA not only on fertilization and cleavage but on pregnancy rate as well ${ }^{12,19}$. In contrast to these studies other authors have not found a statistically significant association ${ }^{20}$. In our study, the fertilization rates and cleavage rates of Ig isotypes were not significantly affected by the method of fertilization. When comparing with ASA negatives as the standard value, IgA and IgG isotypes had higher total fertilization rates $(82.76 \%, 70.75 \%)$ and markedly lower total cleavage rates $(45.82 \%, 49.33 \%)$ than that of ASA negatives (58.54\%, 61.73\%). IgA isotype was observed as having the highest fertilization rate and the lowest cleavage rate. It also shows that IgA isotype has the lowest pregnancy rate $(10.53 \%)$. This suggests that $\operatorname{IgA}$ isotype may have the most deleterious clinical effects on cleaving embryos.

Concerning the relationship between the Ig binding sites and fertilization, Bronson et al., observed that sera containing ASA directed primarily against the sperm head diminished the percentage of oocytes penetrated by sperm and the number of sperm penetrated per oocyte ${ }^{21}$. Studies by Witkin et al., Yeh et al. and Bohring et al., published that antibodies reacting with sperm tail and head could decrease drastically the sperm fertilizing potential ${ }^{11,18,22}$.

Our analysis shows a marked reduction on cleavage rate when ASA was bound to head and significant reduction $(\mathrm{P}$-value $=0.03)$ when bound to midpiece+tail of the sperm surface with that of ASA negatives in standard IVF. Head status of spermatozoa long has been assumed to play a key role in fertilization because of sperm's plasma membrane receptors and its internal nuclear content. The midpiece+tail on the other hand may have high density of ASA on spermatozoa surface which affect cleavage rate. Our study also shows that when ICSI was performed on oocytes, there was no significant difference in cleavage rates among different locations compared to ASA negatives. This is supported by numerous other studies which have shown that ICSI as an effective method of treatment in immunoinfertility ${ }^{23,24,25}$. This technique bypasses most, if not all, of the steps in conception that are adversely affected by antisperm antibodies in human infertility ${ }^{26}$.

Concerning the pregnancy rate with the site of binding of ASA, our data demonstrates that most pregnancies $(n=5)$ were observed with tail bound ASA. Interestingly, no pregnancies were observed with head bound ASA. A single pregnancy was achieved in ASA bound to the whole surface of the sperm with ICSI as 
the method of fertilization. Though the numbers of pregnancies were small to statistically define, it is suggested that head bound ASA may have deleterious post fertilization effects on developing pre implantation embryo. The negative effects of ASA on implantation failure have been reported in several other studies ${ }^{15}$. Moreover, these studies even show impaired pregnancy rates and increased miscarriage rates among ASA positives ${ }^{12,15}$. Nevertheless, more studies with large number and control groups need to be conducted in this regard to achieve conclusive data. The incidence of ASA in our study population was $20.87 \%$. This corresponds with other published literature ${ }^{2}$. Hence, it is observed that infertility due to presence of ASA is relatively rare, but not so uncommon. The question that needs to be answered is should one wait for failed fertilization or repeated unsuccessful treatment procedures to perform tests for ASA retrospectively? This study supports that always it is advisable to detect the presence of ASA at an early stage in infertile workup, for a small individual cost, relative to the total cost for repeated unsuccessful procedures and also to save on time. It is proper time to consider whether detection of ASA should be included in routine IVF test protocols and at early stage in general infertile workup as well.

\section{Conclusion}

Some ASA cause infertility or contribute significantly to infertility in humans. ASA reduce the cleavage rate of embryos significantly. Couples having the IgA isotype posses the lowest cleavage rate as well as the pregnancy rate. Presence of ASA on head region or more than one region of the spermatozoa reduce cleavage rate of oocytes significantly in standard IVF. Head bound ASA appear to have most deleterious effects on early embryonic development and hence pregnancy rate. The development of ICSI has circumvented almost all of the mechanisms of action of ASA. However, ICSI is an invasive, expensive procedure with some risk issues. A significant step forward in this field would be the identification of specific antigens that when bound by ASA would specifically affect fertility. This distinction could then lead to changes in treatment of ASA positives and a more individualized approach to therapy saving time, money and emotional stress of infertile couples.

\section{Acknowledgements}

This study was carried out with the financial support from National Science Foundation, Sri Lanka - Research Grant No. RG/2007/HS/02.

\section{References}

1. Li TS. Sperm immunology, infertility and fertility control. Obstet Gynecol. 1974; 44: 607-23.

2. Lee R, Goldstein M, Ullery BW, Witkin SS, et al. Value of serum antisperm antibodies in diagnosing obstructive azoospermia. Journal of Urology 2009; 181: 264-9.

3. Bronson RA. Antisperm antibodies: a critical evaluation and clinical guidelines. Journal of Reprod Immunology 2000; 45: 159-83.

4. Fu GB, Oian LX, Cui YG, Xuan HB, Zhu JG, Zhang W. Antisperm antibodies induced by testicular torsion and its influence on testicular function. Zhonghua Nan Ke Xue 2006; 13: 63-9.

5. Kutteh WH, Blackwell RE, Gore H, Kutteh CC, et al. Secretory immune system of the female reproductive tract II Local immune system in normal and infected fallopian tube. Fertility and Sterility 1990; 54: 51-5.

6. Livi C, Versary L, Pratesi S, Buzzoni P. Does intraperitoned insemination in the absence of prior sensitization carry with it a higher risk of subsequent immunity to sperm? Fertility and Sterility 1990; 53: 137-42.

7. Fernando DMS. The detection of antisperm antibodies in the male partners of infertile couples; a protocol of sperm preparation for enhancing sperm quality. A thesis for the degree of Doctor of Philosophy, University of Colombo 2001.

8. Clarke GN, Bourne H, Baker HW. Intracytoplasmic sperm injection for treating infertility associated with sperm autoimmunity. Fertil Steril 1997; 68: 112-7.

9. Francavilla F, Santucci R, Barbonetti A, Francavilla S. Naturally occurring antisperm antibodies in men: interference with fertility and clinical implications. An update. Frontiers in Bioscience 2007; 12: 2890-911.

10. Mazumdar S, Levine AS. Antisperm antibodies: etiology, pathogenesis, diagnosis and treatment. Ferility and Sterility 1998; 70: 799-810.

11. Yeh WR, Acosta AA, Seltman HJ, et al. Impact of immunoglobulin isotype and sperm surface location of antisperm antibodies on fertilization in vitro in the human. Fert Steril 1995; 63: 1287-92.

12. Vazquez-Levin MH, Notrica HA, Polak de Fried E. Male immunologic infertility: sperm performance on in vitro fertilization. Fert Steril 1997; 68: 675-81.

13. Bohring C. The role of antisperm antibodies during fertilization and their possible role in immunological infertility. American Journal of Reproductive Immunology 2002; 48: 134.

14. Shibahara H, Shiraishi Y, Hirano Y, Suzuki T, Takamizawa $\mathrm{T}$, Suzuki M. Diversity of the inhibitory effects on fertilization by anti-sperm antibodies bound to the surface of ejaculated human sperm. Human Reproduction 2003; 18: 1469-73. 
15. Cline AM, Kutteh WH. Is there a role of autoimmunity in implantation failure after in vitro fertilization? Curr Opin Obstet Gynecol. 2009; 21: 291-5.

16. Liu DY, Clarke GN, Baker HW. Inhibition of human spermzona pellucida and sperm-oolemma binding by antisperm antibodies. Fertil Steril 1991; 55: 440-4.

17. Naz RK. Effects of antisperm antibodies on early cleavage of fertilized ova. Biology of Reproduction 1992; 46: 130-9.

18. Witkin SS, Viti D, David SS, et al. Relation between antisperm antibodies and the rate of fertilization of human oocyte in vitro. J Assist Reprod Genet 1992; 9: 9-13.

19. Chang TH, Jih MH, Wu TC. Relationship of sperm antibodies in women and men to human in vitro fertilization, cleavage and pregnancy rate. Am J Reprod Immunol. 1993; 30: 108-12.

20. Clarke GN, Baker HW. Lack of association between sperm antibodies and recurrent spontaneous abortion. Fertil Steril 1993; 59: 463-4.

21. Bronson RA, Cooper GW, Rosenfeld DL. Sperm-specific isoantibodies and autoantibodies inhibit the binding of human sperm to the human zona pellucida. 1982; 38: 724-72.
22. Bohring C, Klepper L, Krause W. Localization of binding sites of naturally occurring antisperm antibodies on human spermatozoa by immunofluorescence. Andrologia 2004; 36: 286-90.

23. Check ML, Check JH, Katsoff D, Summers-Chase D. ICSI as an effective therapy for male factor with antisperm antibodies. Arch Androl. 2000; 45: 125-30.

24. Lombardo F, Gandini L, Lenzi A, Dondero F. Antisperm immunity in assisted reproduction. J Reprod Immunol. 2004; 62: 101-9.

25. Shibahara H, Shiraishi Y, Suzuki M. Diagnosis and treatment of immunologically infertile males with antisperm antibodies. Reproductive Medicine and Biology 2005; 4: 133-41.

26. Rumke P, Renckens CN, Bezemer PD, van Amstel N. Prognosis of fertility in women with unexplained infertility and sperm agglutinins in the serum. Fertil Steril 1984; 42: 561-7.

27. World Health Organization. WHO laboratory manual for the examination of human semen and sperm cervical mucus interaction 1999, Fourth edition, Cambridge University Press. 